\title{
Connective Tissue of Villus
}

National Cancer Institute

\section{Source}

National Cancer Institute. Connective Tissue of Villus. NCI Thesaurus. Code C34133.

A type of fibrous tissue that provides cohesion and support to the intra-villus structures, and constitutes the bulk of the villus. 